\title{
Research Staff Perspectives on Cancer Clinical Trials and Barriers to Recruitment: A Qualitative Research
}

\author{
Amany R. Keruakous ${ }^{1,} 2$, Silas Day ${ }^{2}$, Kenny Garcia-Ramiu ${ }^{2}$, Melissa Yarbrough ${ }^{2}$, Adam S. Asch ${ }^{2}$ \\ 1. Hematology and Medical Oncology, Augusta University Medical College of Georgia, Augusta, USA 2. Hematology \\ and Medical Oncology, University of Oklahoma Health Sciences Center, Oklahoma City, USA
}

Corresponding author: Amany R. Keruakous , amany.keruakous@gmail.com

\section{Abstract \\ Background}

Clinical trials are key elements of the processes that account for many of the recent advances in cancer care. Unfortunately, they are becoming more challenging to conduct. Furthermore, a large number of clinical trials in oncology close early due to poor accrual. To identify opportunities for continued improvement in clinical trial enrollment, we sought to identify the obstacles encountered by our clinical trial research staff in these activities.

\section{Methods}

This is a prospective qualitative study, using Grounded Theory Methodology that was concluded at Stephenson Cancer Center (SCC). SCC has been the lead accruer to National Cancer Institute-Lead Academic Participating Sites (NCI-LAPS) trials over the past three years, and in addition, fields investigator-initiated and industry-sponsored trials.

We conducted a survey of our research staff including all research nurses and disease site coordinators who participate in recruitment, screening, consenting, data collection, and compliance for interventional clinical trials. We then performed a follow-up meeting with our research coordinators to clarify responses. The study objectives were to highlight common barriers to recruiting adult cancer patients, encountered by research coordinators from all disease sites and to propose effective solutions to identified barriers.

\section{Results}

We are reporting our results of investigating barriers to clinical trials enrollment from a new perspective. The most commonly reported obstacles for clinical trials enrollment from our research staff's perspective were categorized into five themes: clinical trials protocol, communication barriers and cultural beliefs, financial barriers, patients' comorbidities and performance status, and physicians' commitment.

Review began 08/03/2021 Review ended 08/09/2021 Published 08/15/2021

(๑) Copyright 2021

Keruakous et al. This is an open access article distributed under the terms of the Creative Commons Attribution License CC-BY 4.0., which permits unrestricted use, distribution, and reproduction in any medium, provided the original author and source are credited.

\section{Conclusions}

Although assessing barriers encountered by clinical research staff is an infrequently used metric for improving clinical trial enrollment, it provides an important perspective in the field. Implementing interventions to improve clinical trial feasibility and accrual is critical to improving cancer care.

Categories: Oncology, Quality Improvement, Public Health

Keywords: cancer clinical trials, barriers to clinical trials enrollment, interventions to improve clinical trials accrual, quality improvement, research staff perspective

\section{Introduction}

Clinical trials provide the foundation on which advances in cancer therapies are built, but so much stands in the way. While clinical trial treatment is considered on-guideline and may result in a lower financial burden to the patient, the national average of cancer patients accrued to clinical trials remains only $2-4 \%$ [1,2]. Poor accrual of cancer patients in cancer clinical trials has been a chronic problem in academia as well as community-based practices [3-6]. Furthermore, a large number of clinical trials in oncology close early due to poor accrual. In an analysis of clinical trials for adults registered on Clinicaltrials.gov, researchers found that about $20 \%$ of the trials fail to accrue for various reasons [7].

Reasons reported to be common obstacles for accrual in clinical trials are often investigated independently a) financial barriers [8], b) patient-reported factors [9,10], c) physician-related factors [11], d) barriers encountered in selected patient population [12-16], or e) selected disease sites [17,18]. Understanding the etiology of low patient accrual is necessary to improve participation in clinical trials. Researchers have 
attempted to identify the barriers to clinical trial enrollment by questioning individuals eligible for participation in clinical trials $[9,19]$, physicians involved $[10,20,21]$, or by exploring lessons from failed accruals [22].

The Stephenson Cancer Center, the only National Cancer Institute (NCI)-designated cancer center in the state of Oklahoma, has led accrual to NCI-Lead Academic Participating Sites (LAPS) trials over the past four years. The Stephenson Cancer Center also fields numerous investigator-initiated and industry-sponsored trials. In this study, we sought to identify common obstacles for trial accrual from a new perspective. We surveyed our clinical research staff-- specifically our research coordinators, who are the patient's primary point of contact in the process of patients' recruitment, screening, consenting, data collection, and compliance (Appendices).

The purpose of this study is to a) highlight common barriers to recruiting adult cancer patients encountered by research coordinators across multiple disease sites, and b) propose effective solutions to the identified barriers.

This article was previously published as a preprint article: Keruakous AR, Day S, Garcia-Ramiu KD, Yarbrough M, Asch AS: Research staff perspectives on cancer clinical trials and barriers to recruitment: a qualitative research. 18 March 2021, PREPRINT (Version 1) available at Research Square [https://doi.org/10.21203/rs.3.rs-322279/v1]

\section{Materials And Methods Study design and participants}

This study is a prospective qualitative study, using Grounded Theory Methodology [23,24]. Given the lack of data on barriers encountered by research staff members during the patient's enrollment process in clinical trials, we thought that the qualitative method is appropriate for the study as we are exploring a new perspective by using inductive reasoning starting with data collection and interpretation to construct a theory.

The grounded theory method was felt to be fit for the research question as it addresses their experience and interactions with the patients to identify and categorize barriers to clinical trial enrollment to develop a theory from the analysis grounded in the data provided.

To achieve purposive sampling, we choose participants who are working in a direct relationship with cancer patients from all disease sites and serving a wide diversity of patients. Thus, we focused on the research staff involved in interventional clinical trials in this manner. Participants for this qualitative study were approached via email as we distributed a formal survey that asked open-ended questions to procure frequently encountered obstacles that survey participants meet during their daily interactions with our patients

The number of participants was determined by reaching a point of data saturation (i.e. no more new themes emerging); we then performed follow-up meetings with the respondents using semi-structured interviews which were guided by topic guides and adjusted based on the initial analysis or their email responses which were helpful to clarify and add richness to the data collected.

\section{Data collection}

Data were collected through a survey that was sent via e-mail to describe the following: 1) the disease site they are primarily involved, 2) what is their role in clinical trials (data management, compliance, etc.), 3) what percentage of screened patients decline participation in the clinical trial, 4) what are the most common reasons that patients decline participation, 5) what are the main obstacles to enrollment, and 6) additional comments (where they had the opportunity to describe any additional information and suggestions, in addition to the semi-structured interviews).

\section{Data analysis}

De-identified surveys and follow-up transcribed meetings were collected in one software. Data were categorized into codes creating a codebook to ensure inter-coding agreement. Emerging codes were then discussed and agreed upon by team members. Emerging codes led to themes. The process of defining and refining the themes was continuous throughout the data analysis. Selected research coordinators were asked to review the data collected and the manuscript to ensure confirmability.

\section{Results}

\section{Sample}

Twenty-one surveys were received from research coordinators at different disease sites. Of these 21 , six surveys were from gynecologic malignancies, five from breast oncology, three from lung and head/neck 
malignancies, four from malignant hematology, two from gastrointestinal malignancies, and one from genitourinary malignancies.

\section{Themes}

Five main themes important to clinical trial enrollment were identified: clinical trials protocol, communication barriers and cultural beliefs, financial barriers, patients' comorbidities and performance status, and physicians' commitment. Below we will describe the themes.

\section{Clinical Trial Protocol}

A common obstacle encountered by our research coordinators pertains to the clinical trial schema and protocol requirements. Participants frequently reported that the inclusion and exclusion criteria are often overly strict. Furthermore, it was noted that patients commonly tend to refuse to participate in clinical trials if additional biopsies are required.

Also, frequent laboratory testing and office visits often drive patients away from participation in clinical trials because of their concern of affecting their work schedule and increase the time off work to participate in such activities, especially if they live at a distance from the treating institution. Patients prefer a more flexible approach to treat their condition and preserve their quality of life.

Communication Barrier and Cultural Beliefs

Frequently reported, the communication barrier has been one of the difficult obstacles to overcome. If the patient is speaking a non-English language, commonly Spanish, that often excludes them from participating in clinical trials. The absence of language-appropriate consent or the need to requesting institutional review board (IRB) approval of Spanish informed consent delays the enrollment procedure. The urgency to start the treatment makes waiting to participate in the clinical trial an inferior option for the majority of our patients.

Cultural beliefs linked to the fear of receiving an investigational drug or a placebo are commonly encountered. Also, the common conception of being treated as a "lab rat" while participating in a clinical trial usually exists as a barrier for accrual. These cultural beliefs and misconceptions have also led to the underrepresentation of racial minorities in cancer clinical trials.

Financial Burden

Patients refuse to participate in the clinical trial due to the concern of the additional financial implications that might fall with the additional office visits and laboratory testing, let alone arranging transportation for those visits may create an added barrier. Additionally, the legitimate concern of having extra days off work to accommodate the clinical trial office visits and laboratory testing, adding yet another layer of financial burden.

\section{Patients' Status}

Disease burden affects the patients' general performance status and subsequently excludes them from clinical trials. This barrier can often be counterintuitive to the patient's ultimate well-being. As performance status limitations are often directly or indirectly related to their underlying malignancy, and in such settings, treating the underlying malignancy serves to improve their energy level and performance status. Also, comorbidities remain a common exclusion criterion.

\section{Physician Commitment}

Explaining the science behind the investigational agent, its mechanism of action, and the study hypothesis as well as disclosing benefit/risk balance while incorporating the financial burden and time commitment is crucial for clinical trial screening. Due to the level of confidence and trust built between the primary oncologist and the patients/their families, the clinical trial is best introduced and explained by the treating physician, which requires physician enthusiasm and commitment as well as open communication with the research team.

Clinical Trials Challenges in the Midst of a Pandemic

During the COVID-19 pandemic, our research staff noted continued interest in clinical trials. However, patients express concerns over multiple in-person visits required on trial. Lack of education surrounding risks of admission vs risks of immunosuppression needs to be addressed. Also, screen failures have become more prominent throughout the pandemic as patients delay medical care until the situation is urgent. This leads to a worsened disease burden, worsened performance status, organ dysfunction which disqualifies 


\section{Cureus}

them from participation in many clinical trials.

Illustrative quotes are provided in Table 1.

\begin{tabular}{|c|c|c|}
\hline Themes & Codes & Quoted responses \\
\hline $\begin{array}{l}\text { Clinical trial } \\
\text { protocol }\end{array}$ & $\begin{array}{l}\text { - Frequent } \\
\text { laboratory testing } \\
\text { and office visits. - } \\
\text { Treatment } \\
\text { schedule and time } \\
\text { commitment. - } \\
\text { Strict } \\
\text { inclusion/exclusion } \\
\text { criteria. -Additional } \\
\text { biopsy } \\
\text { requirements. }\end{array}$ & $\begin{array}{l}\text { "Patients often find that: a) Office visits are too much b) Laboratory tests are too frequent in between } \\
\text { office visits c) Treatment schedules interfere with their work schedule hence affecting the quality of life } \\
\text { and preclude financial burden" "Patients live too far away and would prefer a less regimented treatment } \\
\text { which provides more logistical wiggle room" "Strict Inclusion/Exclusion has been the main barrier to } \\
\text { enrollment" "Mandatory tissue requirements of certain specifications often make patients reluctant to } \\
\text { participate" }\end{array}$ \\
\hline $\begin{array}{l}\text { Communication } \\
\text { barrier and } \\
\text { Cultural beliefs }\end{array}$ & $\begin{array}{l}\text {-Language barrier - } \\
\text { Refusal to be on } \\
\text { an investigated } \\
\text { subject -Concerns } \\
\text { about the risks } \\
\text { with an } \\
\text { investigational } \\
\text { agent }\end{array}$ & $\begin{array}{l}\text { "Patients often are not willing to try investigational drugs" "Patients decline is due to the fear of being } \\
\text { experimented on" "Patients have the cultural belief that if they are on a clinical trial, they are being } \\
\text { treated as a guinea pig or a lab rat and refuse to listen about what the trial entails" "Patients do not want } \\
\text { to take the risk of being on placebo arm and would rather standard treatment" "whenever we have not an } \\
\text { English speaker patient, we have difficulty enrolling them on a clinical trial even if they are cooperative } \\
\text { due to lack of foreign language informed consent" }\end{array}$ \\
\hline $\begin{array}{l}\text { Financial } \\
\text { barriers }\end{array}$ & $\begin{array}{l}\text { - Financial burden } \\
\text { of additional } \\
\text { laboratory testing } \\
\text { and office visits. - } \\
\text { Burden of extra } \\
\text { days off to } \\
\text { accommodate the } \\
\text { trial protocol }\end{array}$ & $\begin{array}{l}\text { "A lot of times, patients have expectations that all treatments/procedures while on the study are provided } \\
\text { by the study, they get quite anxious with costs of the additional laboratory testing and the transportations } \\
\text { needed" "The fear of missing extra days off work to follow the study treatment schedule and office visits" }\end{array}$ \\
\hline Patients' status & $\begin{array}{l}\text {-Performance } \\
\text { status - } \\
\text { Comorbidities }\end{array}$ & $\begin{array}{l}\text { "Most of the clinical trials limit inclusion criteria based on performance status which might be affected } \\
\text { due to the disease burden" "Declining physical condition due to disease burden excludes patients" } \\
\text { "Comorbidities is often what excludes patients from participating in clinical trials" }\end{array}$ \\
\hline $\begin{array}{l}\text { Physician } \\
\text { commitment }\end{array}$ & $\begin{array}{l}\text {-Building a } \\
\text { foundation of } \\
\text { confidence. - } \\
\text { Appropriately } \\
\text { introducing the } \\
\text { research team. }\end{array}$ & $\begin{array}{l}\text { "The physicians doing a good job of rationalizing why patients are ideal candidates for a clinical trial prior } \\
\text { to the research nurse/coordinator going in for the consent" "The physician is the person our patient most } \\
\text { relies on to make informed decisions on their healthcare" "Building a foundation of confidence for the } \\
\text { clinical trial and the research" }\end{array}$ \\
\hline $\begin{array}{l}\text { Clinical trials } \\
\text { challenges in } \\
\text { the midst of a } \\
\text { pandemic }\end{array}$ & $\begin{array}{l}\text {-Delays to seek } \\
\text { medical attention } \\
\text { leads to more } \\
\text { screening failures - } \\
\text { Continued interest } \\
\text { but lacking } \\
\text { education - } \\
\text { Implementing } \\
\text { telemedicine visits }\end{array}$ & $\begin{array}{l}\text { "...have not seen a decreased interest in clinical trial enrollment throughout the pandemic" "Clinical trial } \\
\text { infrastructure has been hit during the pandemic" "Patients seem to be more concerned about contracting } \\
\text { COVID at the hospital than in the community" "Patients are not seeking healthcare as soon as they } \\
\text { normally would sue to the pandemic" "More screen failures as patients' diseases have progressed to the } \\
\text { point their performance status or hepatic function is no longer compatible with the clinical trial" } \\
\text { "Accommodate some telemedicine visits for trial patients as well as continue to provide them a safe face } \\
\text { to face visit as well with universal masking, screening at every entrance and limited visitors" "Re-evaluate } \\
\text { what procedures and visits in clinical trials are "unnecessary" in hopes of minimizing the exposure risk for } \\
\text { our patients" }\end{array}$ \\
\hline
\end{tabular}

TABLE 1: Selected quotes illustrating themes and codes

\section{Discussion}

Identifying common obstacles for clinical trial enrollment is a key to improving the clinical trial accrual rate, therefore improving the advancement in cancer treatments [25]. We explored this aspect from our research staff perspective. We aimed to identify barriers to clinical trials accrual and propose potential interventions 
in future trials protocols that would improve the accrual rates and facilitate patients' enrollment and commitment in clinical trials.

The complexity of clinical trials protocol is a reported obstacle by our research coordinators and other studies [26]. Clinical trial protocols that include simple schema, minimizing office visits, and frequency of laboratory testing would be more feasible for patients to participate in clinical trials. If frequent laboratory testing is necessary for investigational drug monitoring, utilizing home health care services for eligible patients to perform blood draws for required laboratory testing, as well as utilizing virtual care visits to follow up on patients' tolerance; would facilitate patients' commitment to clinical trials and eliminate the financial burden of frequent transportation.

Although industry-sponsored clinical trials offer travel reimbursement programs, having this implemented universally for all trials would also aid in recruitment. Utilizing social workers' services to help with transportation aids that are offered by patients' primary insurance should also be considered. Other tools to improve patients' accrual are to broaden the eligibility criteria considering the patients' comorbidities and performance status, as well as minimize additional biopsies required for accrual.

Cultural beliefs are often non-modifiable; however, cultural influences must be considered when recruiting for clinical trials. Encouraging the physicians and research staff to use basic or simple terms in explaining the clinical trials and their purpose while simultaneously addressing patients' concerns, and emphasizing that participating in a clinical trial does not entail being an experimental subject [27,28]. It is noted that despite having a high education level, $75 \%$ of clinical trials participants did not have enough basic research knowledge to make a decision about joining a cancer clinical trial [29]. Community organizations educating the public about cancer research may be the key to enhancing patient enrollment [30].

A language barrier is encountered by one-third of cancer patients as determined by Surveillance, Epidemiology, and End Results (SEER) database statistics review 2000-2017. The majority of these patients are Spanish speaking, therefore having a Spanish informed consent form with every clinical trial protocol suffices as a measure that would efficiently facilitate non-English speakers to participate in clinical trials. At the same time, it demonstrates to that specific patient population that their participation in clinical trials is important in furthering cancer research.

Finally, delivering the best care for cancer patients in general as well as cancer clinical trials has been challenging through the COVID-19 pandemic. Balancing the risks of treatment procedures, its potential complications, and exposure to COVID-19, using the ultimate safety measures following the US Centers for Disease Control and Prevention (CDC) guidelines is crucial. Re-evaluating the infrastructure of clinical trial operations by revising procedures and modifying required visits to minimize exposure should be widely implemented.

Utilizing a qualitative research method using open-ended questions could limit the study, as participants have more control over the content of the data collected, making the data not verifiable objectively against the scenarios stated by the respondents. Simultaneously, this adds another value as it emphasizes patterns that can be hard to see, due to the breadth of emotions, reactions, and responses from study participants.

The research is based more on the opinion and judgment of our research staff rather than on measurable outcomes, and it does not investigate causality, but it does provide indications of possible directions for academic and sponsored clinical trials teams to improve the feasibility of clinical trials and bolster the potential accrual efforts that could be implemented.

\section{Conclusions}

Assessing barriers encountered by clinical research staff is an infrequently used metric for improving clinical trial enrollment, but provides an important perspective. This qualitative study is reporting a new perspective for barriers to clinical trials accrual encountered by our research staff coordinators. The main five themes reported by our research staff as commonly encountered obstacles to clinical trails enrollment are: the complexity of clinical trials protocol, communication barriers and cultural beliefs, financial barriers, patients' comorbidities and performance status, and physicians' commitment.

In our study, some obstacles are inherent in our patient populations, others appear to be actionable. We proposed possible measures that could be implemented into clinical trial protocols to facilitate patients' accrual. The complexity of clinical trial protocols and the increasingly strict inclusion/exclusion criteria are issues that will require consideration and action at the level of the cooperative groups and industry. Additionally, accommodating communications and cultural barriers with increasing institutional and physicians' commitment could overcome negative bias regarding clinical trials, and enhance recruiting underrepresented minorities.

\section{Appendices}




\section{Research staff survey}

Dear Research staff,

Please share your opinion on obstacles to clinical trial enrollment.

I'm trying to track factors that potentially affect patient enrollment into cancer clinical trials; and as you're all intimately involved with screening and consenting patients here at SCC, I would be very grateful to get your feedback.

1. Is there a disease site in which you are primarily involved?

2. What's your role in clinical trials (nurse, coordinator, data, compliance, etc)?

3. What are the most common reasons that patients decline participation?

4. What are the main obstacles to enrollment (language, finances, overly strict inclusion/exclusion criteria, cultural issues, etc.)

5. Any additional comments on the process would also be appreciated.

Thanks to all of you for your time and your help to improve patients' enrollment in cancer clinical trials.

\section{Additional Information \\ Disclosures}

Human subjects: Consent was obtained or waived by all participants in this study. University of Oklahoma, IRB issued approval 13046. Project Title: Research Staff Perspectives on Cancer Clinical Trials and Barriers to Recruitment: A Qualitative Research On behalf of the Institutional Review Board (IRB), I have reviewed the Determination of Human Research Worksheet for the above-referenced project. Based on the information provided, I have determined this does not meet the criteria for human subjects research. No additional action is required by the IRB. If you have questions about this notification or using iRIS, contact the HRPP office at (405) 271-2045 or irb@ouhsc.edu. Sincerely, Laura L. Holman, MD, Chair Institutional Review Board. Animal subjects: All authors have confirmed that this study did not involve animal subjects or tissue. Conflicts of interest: In compliance with the ICMJE uniform disclosure form, all authors declare the following: Payment/services info: All authors have declared that no financial support was received from any organization for the submitted work. Financial relationships: All authors have declared that they have no financial relationships at present or within the previous three years with any organizations that might have an interest in the submitted work. Other relationships: All authors have declared that there are no other relationships or activities that could appear to have influenced the submitted work.

\section{References}

1. Rimel BJ: Clinical trial accrual: obstacles and opportunities. Front Oncol. 2016, 6:103. 10.3389/fonc. 2016.00103

2. Unger JM, Cook E, Tai E, Bleyer A: The role of clinical trial participation in cancer research: barriers, evidence, and strategies. Am Soc Clin Oncol Educ Book. 2016, 35:185-98. 10.1200/EDBK_156686

3. Dilts DM, Sandler AB, Cheng SK, et al.: Steps and time to process clinical trials at the Cancer Therapy Evaluation Program. J Clin Oncol. 2009, 27:1761-6. 10.1200/JCO.2008.19.9133

4. Knelson LP, Cukras AR, Savoie J, et al.: Barriers to clinical trial accrual: perspectives of community-based providers. Clin Breast Cancer. 2020, 20:395-401.e3. 10.1016/j.clbc.2020.05.001

5. Go RS, Frisby KA, Lee JA, et al.: Clinical trial accrual among new cancer patients at a community-based cancer center. Cancer. 2006, 106:426-33. 10.1002/cncr.21597

6. Lara PN Jr, Higdon R, Lim N, et al.: Prospective evaluation of cancer clinical trial accrual patterns: identifying potential barriers to enrollment. J Clin Oncol. 2001, 19:1728-33. 10.1200/JCO.2001.19.6.1728

7. Schroen AT, Petroni GR, Wang H, et al.: Preliminary evaluation of factors associated with premature trial closure and feasibility of accrual benchmarks in phase III oncology trials. Clin Trials. 2010, 7:312-21. 10.1177/1740774510374973

8. Largent EA, Lynch HF: Addressing financial barriers to enrollment in clinical trials . JAMA Oncol. 2018, 4:913-4. 10.1001/jamaoncol.2018.0492

9. Mills EJ, Seely D, Rachlis B, et al.: Barriers to participation in clinical trials of cancer: a meta-analysis and systematic review of patient-reported factors. Lancet Oncol. 2006, 7:141-8. 10.1016/S1470-2045(06)70576-9

10. Mahmud A, Zalay O, Springer A, Arts K, Eisenhauer E: Barriers to participation in clinical trials: a physician survey. Curr Oncol. 2018, 25:119-25. 10.3747/co.25.3857

11. Embi PJ, Jain A, Clark J, Bizjack S, Hornung R, Harris CM: Effect of a clinical trial alert system on physician participation in trial recruitment. Arch Intern Med. 2005, 165:2272-7. 10.1001/archinte.165.19.2272

12. Denson AC, Mahipal A: Participation of the elderly population in clinical trials: barriers and solutions . Cancer Control. 2014, 21:209-14. 10.1177/107327481402100305

13. Fern LA, Whelan JS: Recruitment of adolescents and young adults to cancer clinical trials--international comparisons, barriers, and implications. Semin Oncol. 2010, 37:1-8. 10.1053/i.seminoncol.2010.04.002 
14. Fern LA, Taylor RM: Enhancing accrual to clinical trials of adolescents and young adults with cancer . Pediatr Blood Cancer. 2018, 65:e27233. 10.1002/pbc.27233

15. Mouton CP, Harris S, Rovi S, Solorzano P, Johnson MS: Barriers to black women's participation in cancer clinical trials. J Natl Med Assoc. 1997, 89:721-7.

16. Ford JG, Howerton MW, Lai GY, et al.: Barriers to recruiting underrepresented populations to cancer clinical trials: a systematic review. Cancer. 2008, 112:228-42. 10.1002/cncr.23157

17. Haddad RI, Chan AT, Vermorken JB: Barriers to clinical trial recruitment in head and neck cancer . Oral Oncol. 2015, 51:203-11. 10.1016/j.oraloncology.2014.12.007

18. Melisko ME, Hassin F, Metzroth L, et al.: Patient and physician attitudes toward breast cancer clinical trials: developing interventions based on understanding barriers. Clin Breast Cancer. 2005, 6:45-54. 10.3816/CBC.2005.n.008

19. Schaeffer MH, Krantz DS, Wichman A, Masur H, Reed E, Vinicky JK: The impact of disease severity on the informed consent process in clinical research. Am J Med. 1996, 100:261-8. 10.1016/S0002-9343(97)89483-1

20. Hudson SV, Momperousse D, Leventhal H: Physician perspectives on cancer clinical trials and barriers to minority recruitment. Cancer Control. 2005, 12 Suppl 2:93-6. 10.1177/1073274805012004S14

21. Benson AB 3rd, Pregler JP, Bean JA, Rademaker AW, Eshler B, Anderson K: Oncologists' reluctance to accrue patients onto clinical trials: an Illinois Cancer Center study. J Clin Oncol. 1991, 9:2067-75. 10.1200/TCO.1991.9.11.2067

22. Tognoni G, Alli C, Avanzini F, et al.: Randomised clinical trials in general practice: lessons from a failure . BMJ. 1991, 303:969-71. 10.1136/bmj.303.6808.969

23. Thompson CB, Walker BL: Basics of research (part 12): qualititative research. Air Med J. 1998, 17:65-70. 10.1016/S1067-991X(98)90022-0

24. Annells M: Grounded theory method, part i: within the five moments of qualitative research . Nurs Inq. 1997, 4:120-9. 10.1111/j.1440-1800.1997.tb00085.x

25. Crombie IK, McMurdo ME, Irvine L, Williams B: Overcoming barriers to recruitment in health research: concerns of potential participants need to be dealt with. BMJ. 2006, 333:398. 10.1136/bmj.333.7564.398

26. Vastola ME, Yang DD, Muralidhar V, Mahal BA, Lathan CS, McGregor BA, Nguyen PL: Laboratory eligibility criteria as potential barriers to participation by black men in prostate cancer clinical trials. JAMA Oncol. 2018, 4:413-4. 10.1001/jamaoncol.2017.4658

27. Somkin CP, Altschuler A, Ackerson L, et al.: Organizational barriers to physician participation in cancer clinical trials. Am J Manag Care. 2005, 11:413-21.

28. Pinto HA, McCaskill-Stevens W, Wolfe P, Marcus AC: Physician perspectives on increasing minorities in cancer clinical trials: an Eastern Cooperative Oncology Group (ECOG) Initiative. Ann Epidemiol. 2000, 10:78-84. 10.1016/S1047-2797(00)00191-5

29. Biedrzycki BA: Research information knowledge, perceived adequacy, and understanding in cancer clinical trial participants. Oncol Nurs Forum. 2011, 38:E291-6. 10.1188/11.ONF.E291-E296

30. Petitte T, Nichols A, Narsavage G: Cultural and environmental enablers and barriers to participation and retention in clinical trials for cancer research. EC Pulmonol Respir Med. 2019, 8:600-6. 